\title{
Study of predictor corrector block method via multiple shooting to Blasius and Sakiadis \\ flow
}

\begin{abstract}
In this paper, a predictor corrector two-point block method is proposed to solve the wellknown Blasius and Sakiadis flow numerically. The Blasius and Sakiadis flow will be modeled by a third order boundary value problem. The main motivation of this study is to provide a new method that can solve the higher order BVP directly without reducing it to a system of first order equation. Two approximate solutions will be obtained simultaneously in a single step by using predictor corrector two-point block method able to solve the third order boundary value problem directly. The proposed direct predictor corrector two-point block method will be adapted with multiple shooting techniques via a three-step iterative method. The advantage of the proposed code is that the multiple shooting will converge faster than the shooting method that has been implemented in other software. The developed code will automatically choose the guessing values in order to solve the given problems. Some numerical results are presented and a comparison to the existing methods has been included to show the performance of the proposed method for solving Blasius and Sakiadis flow.
\end{abstract}

Keyword: Blasius flow; Sakiadis flow; Predictor corrector block method; Multiple shooting technique 
